\begin{tabular}{|c|l|}
\hline Title & $\begin{array}{l}\text { Construction of an infectious cDNA clone for Omsk hemorrhagic fever virus, and characterization of mutations in } \\
\text { NS2A and NS5 }\end{array}$ \\
\hline Author(s) & Yoshii, Kentaro; Igarashi, Manabu; Ito, Kimihito; Kariwa, Hiroaki; Holbrook, Michael R.; Takashima, Ikuo \\
\hline Citation & $\begin{array}{l}\text { Virus Research, 155(1), 61-68 } \\
\text { https://doi.org/10.1016j.virusres.2010.08.023 }\end{array}$ \\
\hline Issue Date & 2011-01 \\
\hline Doc URL & http://hdl.handle.net/2115/45025 \\
\hline Type & article (author version) \\
\hline Additional Information & There are other files related to this item in HUSCAP. Check the above URL. \\
\hline File Information & VR155-1_61-68.pdf \\
\hline
\end{tabular}

Instructions for use 


\title{
Construction of an infectious cDNA clone for Omsk Hemorrhagic fever virus, and characterization of mutations in NS2A and NS5
}

\author{
Kentaro Yoshii ${ }^{1,2}$, Manabu Igarashi ${ }^{3}$, Kimihito Ito $^{3}$, Hiroaki Kariwa ${ }^{1}$, Michael R Holbrook ${ }^{2,4}$, \\ Ikuo Takashima ${ }^{1}$
}

${ }^{1}$ Laboratory of Public Health, Graduate School of Veterinary Medicine, Hokkaido University, Sapporo, Hokkaido 060-0818, Japan

${ }^{2}$ Department of Pathology, Institute for Human Infections and Immunity, University of Texas

Medical Branch, Galveston, Texas 77555, US

${ }^{3}$ Department of Global Epidemiology, Hokkaido University Research Center for Zoonosis Control, Sapporo 001-0020, Japan.

${ }^{4}$ NIAID Integrated Research Facility, Frederick, MD 21702, US

Corresponding author: Dr. Kentaro Yoshii

Postal address: Laboratory of Public Health, Graduate School of Veterinary Medicine, Hokkaido University, kita-18 nishi-9, kita-ku, Sapporo, Hokkaido 060-0818, Japan

Tel/fax: +81-11-706-5213

E-mail: kyoshii@vetmed.hokudai.ac.jp 


\begin{abstract}
Omsk hemorrhagic fever virus (OHFV) is a member of the tick-borne encephalitis serocomplex of flaviviruses, and causes hemorrhagic disease in humans. In this study, an infectious cDNA of OHFV was constructed to investigate the molecular mechanisms involved in OHFV pathogenesis for the first time. Our cDNA clone was capable of producing infectious virus which is genetically identical to the parental Guriev strain, and the recombinant virus showed similar biological properties to the parental virus including growth kinetics and virulence characteristics. While characterizing the cDNAs, fortuitous mutations at NS2A position 46 and NS5 position 836 were found to affect viral production. By using a viral replicon expressing luciferase, it was shown that both of the mutations produced a defect in RNA replication and that the NS5 mutation induced a temperature-sensitive phenotype, indicating the importance of these residues in RNA replication. This infectious cDNA will be a useful tool to study the replication and pathogenesis of OHFV.
\end{abstract}

Keywords: Omsk hemorrhagic fever; flavivirus; infectious cDNA; viral replication. 


\section{Abbreviations}

OHF: Omsk hemorrhagic fever

OHFV: Omsk hemorrhagic fever virus

TBE: tick-borne encephalitis

LGTV: Langat virus

ALKV: Alkhurma virus

KFDV: Kyasanur Forest disease virus

C: core

prM: premembrane

E: envelope

NS: non-structural

HDV-RZ: hepatitis delta virus ribozyme

MOI: multiplicity of infection

RdRp: RNA-dependent RNA polymerase

EM: energy minimization

rms: root mean square

ts: temperature-sensitive

NLS: nuclear localization sequence 


\section{Introduction}

Omsk hemorrhagic fever (OHF) is a disease caused by Omsk hemorrhagic fever virus (OHFV), which belongs to the tick-borne encephalitis (TBE) serocomplex, genus Flavivirus, family Flaviviridae. OHFV was first isolated in 1947 from a human presenting with hemorrhagic fever. OHFV is endemic to a fairly localized region of Siberia within the Omsk and Novosibirsk Oblasts in Russia (Burke and Monath, 2001). OHFV is transmitted via the bite of its primary tick vector, Dermacentor reticulates. The transmission cycle of OHFV involves water voles (Arvicola terrestris) and muskrats (Ondata zibethica), and many other animals within endemic area can be infected with OHFV (Busygin, 2000; Kharitonova and Leonov, 1985).

The TBE complex includes tick-borne encephalitis virus (TBEV), Powassan virus, Langat virus (LGTV), Louping ill virus, OHFV, Alkhurma virus (ALKV), and Kyasanur Forest disease virus (KFDV) (Buchen-Osmond, 2003). Although the TBE complex is largely represented by viruses causing encephalitis, OHFV, ALKV and KFDV are known to cause hemorrhagic disease. Unlike ALKV and KFDV, OHFV causes a hemorrhagic disease in humans with few neurological effects (Burke and Monath, 2001). Human OHFV infection results in fever, headache, myalgia, dehydration, and hemorrhage. The mortality rate for $\mathrm{OHF}$ is estimated to be $0.4 \%-2.5 \%$ (Kharitonova and Leonov, 1985). In the mouse model, OHFV causes disease with few neurological signs compared to neurotropic tick-borne flaviviruses and has also demonstrated significantly different tissue localization indicating potential differences in host cell interactions (Holbrook et al., 2005). However, the specific viral and host response mechanisms involved in OHFV pathogenesis are not well understood.

The flavivirus genome consists of a positive-polarity, single-stranded RNA of approximately 11 $k b$, which encodes three structural proteins, i.e., the core (C), premembrane (prM), and envelope (E) 
proteins, and seven non-structural (NS) proteins (NS1, NS2A, NS2B, NS3, NS4A, NS4B, and NS5), within a single long open reading frame (Chambers et al., 1990). The 5'- and 3'-UTRs have predicted secondary structures that are implicated in viral replication, translation, and packaging of the genomes (Gritsun et al., 1997; Proutski et al., 1997; Rauscher et al., 1997).

Infectious cDNA clones have been generated for multiple flaviviruses, and they provide a useful platform on which to investigate the genetic determinants of flavivirus virulence. There are several reports of infectious cDNA clones for TBE serocomplex, TBEV and LGTV (Campbell and Pletnev, 2000; Gritsun and Gould, 1998; Hayasaka et al., 2004; Mandl et al., 1997; Pletnev, 2001). However, an infectious clone has not been developed for OHFV. In this study, we report the construction and characterization of an infectious cDNA clone of OHFV. Furthermore, while characterizing this cDNA, several fortuitous mutations in NS proteins were shown to attenuate viral replication and reduce virulence in mice.

\section{Material and methods}

\section{1. Cell and virus}

BHK cells were grown in MEM supplemented with 8 \% FBS. OHFV strain Guriev was obtained from the World Reference Collection for Emerging Viruses and Arboviruses (WRCEVA) and used for construction of the infectious cDNA clone.

\section{2. Plasmid constructions}

Total cellular RNA was extracted from OHFV (strain Guriev)-infected BHK cells using Trizol (Invitrogen). OHFV RNA was reverse-transcribed with specific oligonuleotide primers using Superscript II reverse transcriptase (Invitrogen). The fragments of OHFV cDNA were amplified by Platinum Taq polymerase (Invitrogen) using specific oligonucleotide primers (see Fig. 1), resolved 
by gel electrophoresis and purified using the Qiaquick gel extraction kit (Qiagen).

The resulting fragments were digested with restriction endonucleases and cloned into the low copy plasmid pACNR provided by Dr. Peter Mason (Ruggli et al., 1996) as depicted in Fig. 1. The oligonucleotide used to amplify th' $\mathrm{UBR}$ included a T7 promoter recognition site and an additional $G$ preceding the first base of the viral genome. A synthetic oligonucleotide was used to add Cla I site at nucleotides 2436-2441. This silent mutation was engineered to permit the ligation of the fragment containing the structural protein gene. A hepatitis delta virus ribozyme (HDV-RZ)/bacteriophage T7 terminator fragment (Mason et al., 2002) was fused to the 3' end of the viral genome to create synthetic run-off transcripts that contained a 3' terminus identical to the viral RNA. The resulting cloned plasmids (designated as OHF-IC) were isolated by standard techniques and sequences were checked at the University of Texas Medical Branch (UTMB) Protein Chemistry Laboratory and compared to the sequence of the original OHFV Guriev sequence (accession No. AB507800). Sequencing identified three mutations encoding amino acid changes at NS2A-46, NS5-65 and NS5-836.

To repair the mutations found in the OHF-IC plasmids, the fragments between nucleotide 3612-3685 (Hpa I - Sph I site), 7094-8778 (Bam HI - Kpn I site), 9488-10295 (Nde I - Apa I site) were amplified by RT-PCR and subsequently cloned into pCR2.1 plasmid (TA cloning kit; Invitrogen). The virus-specific sequence of each intermediate cloning product was checked by sequence analysis. These intermediate plasmids containing the correct sequence were cut by restriction enzymes described above and used to substitute the regions containing the mutations.

Subgenomic OHFV replicons expressing luciferase gene (OHF-REP-luc (Yoshii and Holbrook, 2009)) were used to analyze the effect of the mutations on viral genome replication. Fragments from the mutated OHF-IC plasmids were amplified using the same primers sets described above. 
The PCR product from the fragments containing mutations was cloned into the replicon plasmid to generate replicons containing the specific mutations found in OHF-IC.

\section{3. RNA synthesis-transfection}

The RNAs were synthesized as described previously (Yoshii et al., 2005). Briefly, the plasmids were prepared for run-off transcription by digestion with $X b a \mathrm{I}$ restriction endonuclease, and the resulting template DNAs were in vitro transcribed using the mMESSAGE mMACHINE T7 Kit (Ambion) in a $20-\mu 1$ reaction mixture that contained an additional $1 \mu 1$ of $20 \mathrm{mM}$ GTP solution. After transcription at $37^{\circ} \mathrm{C}$ for $2 \mathrm{~h}$, the template DNAs were removed by DNase I digestion at $37^{\circ} \mathrm{C}$ for 15 min. The RNA was precipitated using lithium chloride, washed with $70 \%$ ethanol, resuspended in RNase-free water, quantitated by spectrophotometer, and stored at $-80{ }^{\circ} \mathrm{C}$ in aliquots. The synthesized RNA was transfected into BHK cells using TransIT-mRNA (Mirus Bio) by a slight modification of the manufacturer's protocol.

\section{4. Detection of OHF V-antigen (IFA)}

Cells were fixed with 3\% paraformaldehyde, and permeabilized with $0.2 \%$ Triton X-100. After blocking with 2\% BSA, the cells were incubated with Rabbit polyclonal antibodies against TBEV E proteins which is cross-reactive to OHFV E proteins (Yoshii et al., 2004), and then treated with Alexa 555-conjugated anti-rabbit IgG antibodies (Invitrogen). The images were viewed and recorded using fluorescence microscopy.

\section{5. Viral titration and growth curves}

Stock preparations of recombinant OHFV from OHF-IC were produced by passaging the virus harvested from the supernatant of RNA-transfected cells once in BHK cells. The sequence of the RNA of the recovered stock virus was confirmed to be identical to that of the each OHF-IC plasmid 
by sequence analysis after RT-PCR. For titrations, cell monolayers prepared in multi-well plates were incubated with serial dilutions of virus for $1 \mathrm{~h}$, and then overlaid with MEM containing $2 \%$ FBS and $1.5 \%$ carboxymethyl cellulose (CMC; Sigma) and incubated for 5 days. After incubation, the cells were fixed and stained with $0.25 \%$ crystal violet in $10 \%$ buffered formalin. Plaques were counted and expressed as PFU/ml. For growth curves, BHK cells were infected at a multiplicity of infection (MOI) of 0.01 . After virus adsorption for $1 \mathrm{~h}$, the inocula were removed, and the cells were washed with PBS and incubated in MEM containing $2 \%$ FBS. The media was harvested at 24, 48, 72, $96 \mathrm{~h}$ post-infection and stored at $-80^{\circ} \mathrm{C}$.

\section{6. Luciferase assay}

For preparation of cell extracts for luciferase assays, BHK cells were washed with PBS and lysed by the addition of cell culture Reporter lysis buffer (Promega), followed by the incubation of cells at room temperature for $10 \mathrm{~min}$. The cell extracts were then harvested and stored at $-80{ }^{\circ} \mathrm{C}$. Luciferase assays were carried out using Luciferase Assay System (Promega) according to manufacturer instructions, and luminescence was determined using a Microplate Luminometer.

\section{7. Virulence in mice}

Eight-week-old female C57BL/6J mice (Charles River Japan, Inc.) were challenged with 1,000 pfu of each virus subcutaneously in the dorsal region. The physical conditions of the mice were observed and the body weight was measured daily. All procedures were according to the guidelines of the Animal Care and Use Committee of the Hokkaido University.

\section{8. Molecular modeling}

A homology model of OHFV RNA-dependent RNA polymerase (RdRp) domain was constructed based on the crystal structure of the dengue virus RdRp domain (PDB code, 2J7W, 
sequence identity: ca 61\%). MODELLER 9v6 (Eswar et al., 2003) was used for homology modeling of OHFV RdRp domains. After one hundred models of the RdRp domain were generated, a model was chosen by the MODELLER objective function value. After addition of hydrogen atoms, the model was refined by energy minimization (EM) using CHARMm force field with the Discovery Studio 2.1 software package (Accelrys, San Diego, CA). Steepest descent followed by conjugate gradient minimizations was carried out until the root mean square (rms) gradient was less than or equal to $0.05 \mathrm{kcal} / \mathrm{mol} / \mathrm{A}$. The generalized Born implicit solvent model (Still et al., 1990; Tsui and Case, 2000) was used to model the effects of solvation. The molecular model was finally evaluated by using PROCHECK (Laskowski et al., 1993) and VERIFY-3D (Eisenberg et al., 1997). The model structure was displayed by PyMOL (DeLano Scientific LLC) (DeLano, 2002).

\section{Results}

\section{1. The construction of the full-length infectious clone of OHFV}

The overall strategy to construct the full-length infectious clone of OHFV strain Guriev is outlined in Fig 1. The viral RNA was extracted from infected BHK cells, and individual dsDNA fragments were amplified by RT-PCR, as shown Fig 1. The six individual fragments were readily assembled into the low-copy plasmid pACNR, which has been used successfully to construct stable infectious clones in several flaviviruses (Bredenbeek et al., 2003; McElroy et al., 2005; Rossi et al., 2005). The complete genome sequence of this plasmid (designated OHF-IC-ori) was determined and compared with the parent virus. There were 10 nucleotide differences with three resulting in amino acid changes, one in NS2A and two in NS5 (Table 1). An additional nucleotide change was intentionally designed to create a ClaI site ( $\mathrm{C}$ to $\mathrm{G}$ at nt 2439). This restriction site was used for the cloning of the DNA fragment between nt 135 and 2441. The other mutations were caused by PCR steps during the amplification of each fragment. These mutations were repaired by substitution as 
described in Materials and Methods, and the infectious clone of OHFV, which is genetically identical to the parent virus Guriev, was obtained and designated as OHF-IC-pt. These OHF-IC plasmids were found to be stable during transformations into E. coli and large-scale plasmid production. After several bacterial passages, the sequence of the plasmid was identical.

\section{2. Generation of OHFV from infectious clone}

XbaI-linearized OHF-IC template was used for in vitro RNA transcription using T7 RNA polymerase and the resulting full-length OHFV transcripts were transfected into BHK cells. At 5 days post-transfection, the transfected cells were fixed and the expression of virus proteins was analyzed by immunofluorescence assay. Most of the cells transfected with the transcripts from OHF-IC-pt showed a perinuclear signal when stained with an E-specific antibody (Fig 3A). Infectious virus could be harvested from the supernatants of the transfected cells, and a cytopathic effect was observed in these cell cultures. However no infectious virus could be recovered from cells transfected with RNAs from OHF-IC-ori, and no virus antigens were observed in the cells by immunofluorescence assay (Fig 3B). These data indicated two things: the production of viral proteins in the cells transfected with RNAs from OHF-IC-pt was not merely the result of translation of the input RNA, and the mutations in OHF-IC-ori affected the production of viral proteins and infectious viruses.

The growth properties of OHF-IC-pt derived virus and wild-type OHFV were analyzed by monitoring the release of virus after infection. BHK cells were infected at m.o.i. of 0.01 with OHFV. Virus was harvested at $24 \mathrm{~h}$ intervals and the yield was quantified by plaque assay (Fig 4). There was slight difference in the yields of virus at $24 \mathrm{~h}$ post-infection, but the resulting growth curves indicate similar growth properties between the parent virus and recombinant virus from OHF-IC-pt. The slight difference at 24 h post-infection may derive from the quasispecies of the parent virus stock because the virus was not plaque purified. 


\section{3. Characterization of NS mutant}

To determine how the mutations in OHF-IC-ori affected the viral production from the OHFV infectious cDNAs, we prepared several infectious cDNAs that incorporated the mutations identified in OHF-IC-ori (Fig 2). The OHF-IC-NS565-836, OHF-IC-NS565 and OHF-IC-NS5836 plasmids have the NS5 mutations, and OHF-IC-NS2A 46 has the NS2A mutation. Recombinant viruses were recovered from these infectious cDNAs, and the growth curves were generated as described above.

No significant differences were observed between the growth curves of OHF-IC-pt and OHF-IC-NS5 $_{65}$ (Fig 4), indicating that the amino acid difference at NS5 position 65 (L to P) did not affect virus growth in BHK cells. Meanwhile, the growth of OHF-IC-NS2A 46 , OHF-IC-NS5 ${ }_{65-836}$ and OHF-IC-NS5 ${ }_{836}$ was restricted relative to OHF-IC-pt and OHF-IC-NS5 $5_{65}$. These results indicate that the mutations at NS2A position 46 ( $\mathrm{L}$ to H) and NS5 position 836 (D to G) limited the virus growth in cell culture.

To further investigate the effect of the NS2A and NS5 mutations on virus production, we prepared a replicon of OHFV containing the luciferase reporter gene with or without the NS mutations (see Materials and Methods and Fig. 2). These replicon RNAs were transfected into BHK cells, and luciferase activities were measured at $6 \mathrm{~h}$ and 3 days post-transfection. There was no difference between the luciferase activities of the replicons at $6 \mathrm{~h}$ post-transfection (Fig. 5A), indicating that the mutations had no effect on the initial translation of reporter gene from transfected replicon RNA. At 3 days post-transfection, the luciferase activity was lower in the lysate from the replicons with mutations at NS2A position 46 or NS5 position 836 at $37^{\circ} \mathrm{C}$ (Fig. 5B). In contrast, there was no decrease in luciferase activities was detected in the lysates from the replicon with and without the mutation at NS5 position 836 at $30{ }^{\circ} \mathrm{C}$. Luciferase activity in cells transfected with the replicon containing the NS2A-46 mutation remained diminished in cells incubated at 30C. Similar temperature sensitivity of NS5-836 was observed in the virus production at $30{ }^{\circ} \mathrm{C}$ (supplementary 
figure 1). These data indicate that the mutations at NS2A-46 and NS5-836 reduce viral replication by reducing RNA replication and that the NS5-836 mutation produces a temperature-sensitive (ts) defect in RNA replication.

\section{4. Pathogenicity of infectious clone-derived viruses in mouse model}

In our previous studies, we described that laboratory mice infected with OHFV showed clinical signs and pathology similar to reports of human infection (Holbrook et al., 2005; Tigabu et al., 2009). The pathogenicity of recombinant viruses was examined in the mouse model. Five adult C57BL/6 mice were infected subcutaneously with 1,000 p.f.u. of each virus and survival was recorded for 28 days (Fig. 6).

OHF-IC-pt virus which is genetically identical to parental OHFV Guriev strain produced $100 \%$ mortality of mice (mean survival time $13.8 \pm 1.92$ ). All mice showed general signs of illness such as hunched posture, ruffled fur, and general malaise, but did not have significant or consistent indications of neurologic disease. After onset of disease, they showed a sharp decrease in body weight beginning at 9 and 10 dpi. When examined postmortem, all mice had conjunctival suffusion with crusting and some had bowel hemorrhage. These results were consistent with our previous data in which mice infected with parental OHFV Guriev strain had viscerotropic disease with limited signs of neurological disease (100 \% mortality and mean survival time $12.8 \pm 2.49$ )(Tigabu et al., 2009).

The OHF-IC-NS5 65 virus had similar virulence to the OHF-IC-pt virus (100\% mortality and mean survival time $14.2 \pm 1.92$ ) indicating that the amino acid difference at NS5 position 65 did not affect the biological properties of OHFV confirming observations from in vitro studies. However, the OHF-IC-NS2A 46 , OHF-IC-NS5 ${ }_{65-836}$ and OHF-IC-NS5 ${ }_{836}$ virus showed decreased virulence. The OHF-IC-NS5 $5_{65-836}$ and OHF-IC-NS5 ${ }_{836}$ virus killed three mice, but survival time was increased while the OHF-IC-NS2A 46 virus was completely attenuated. The NS5-836 mutation was retained in 
the dead mice inoculated with The OHF-IC-NS5 ${ }_{65-836}$ and OHF-IC-NS5 $5_{836}$ virus. All surviving mice inoculated with the OHF-IC-NS2A 46 , OHF-IC-NS5 $5_{65-836}$ and OHF-IC-NS5 ${ }_{836}$ virus showed no signs of illness and no weight loss. These results indicate that the mutations at NS2A position 46 and NS5 position 836 affect OHFV virulence in association with the lower viral replication. All surviving mice inoculated with OHF-IC-NS2A46, OHF-IC-NS5 ${ }_{65-836}$ and OHF-IC-NS5 836 had neutralizing antibody against OHFV ( 320 in 50\% reduction), indicating that the virus was able to replicate at the initial stage of infection without causing a disease.

\section{Discussion}

This is the first report of generation of an infectious clone of OHFV. Infectious clones can be a valuable tool for studying the molecular biology of virus replication, virus structure, virulence determinants, and vaccine development. There is a common difficulty in the construction of full-length clones of flaviviruses, because the plasmids containing a full-length cDNA of these viruses are often unstable during propagation in E. coli. Therefore, by using low-copy-number plasmids and specific bacterial hosts, stable full-length infectious clones have been developed for several flaviviruses (Gritsun and Gould, 1998; Hayasaka et al., 2004; Kinney et al., 1997; Mandl et al., 1997; Shi et al., 2002; Yamshchikov et al., 2001; Yun et al., 2003). In this study, the low-copy-number plasmid pACNR was used for the construction of the full-length OHFV cDNA. This vector has been used to construct stable infectious clones of several flaviviruses and pestiviruses (Bredenbeek et al., 2003; McElroy et al., 2005; Mendez et al., 1998; Ruggli et al., 1996). The OHF-IC plasmids were stable during passaging in bacteria, indicating that this infectious clone can be useful for genetic manipulations.

The Guriev strain, which was isolated from human blood, was selected to construct a full-length 
infectious clone. We previously demonstrated the similarity between human and murine infection with OHFV (Holbrook et al., 2005; Tigabu et al., 2009). The OHFV-infected mice had no indication of neurological problems, and had conjunctival suffusion that has also been reported in human cases. The recombinant virus which is genetically identical to the parental Guriev strain showed similar biological properties to the parental virus, including growth kinetics and virulence characteristics. These results indicate that an efficient reverse genetics system has been established for OHFV.

Our results have identified two amino acid codon substitutions associated with attenuation of the virus production, i.e., Leu46 to His in the NS2A, and Asp836 to Gly in NS5. These substitutions decreased the efficiency of RNA replication, leading to limited virus propagation and decreased virulence in mice.

Flavivirus NS2A is a small, hydrophobic, membrane associated protein involved in RNA replication. It was reported that NS2A binds with high specificity to the 3' untranslated region (UTR) of viral genomic RNA and to other components of the replication complex (Mackenzie et al., 1998). In addition, NS2A is considered to play roles in modulating the host-antiviral interferon response (Liu et al., 2004; Liu et al., 2006; Liu et al., 2005; Munoz-Jordan et al., 2003) and assembly/ secretion processes of virus particles (Kummerer and Rice, 2002; Leung et al., 2008; Liu et al., 2003). Although the exact membrane topology of NS2A is yet to be determined, NS2A of OHFV has been predicted to span the membrane of the endoplasmic reticulum five times by several transmembrane domain prediction programs (TMHMM (Krogh et al., 2001), and TMpred (Hofmann and Stoffel, 1993)). The Leu46 residue in NS2A is located in the conserved hydrophobic residues of the predicted $2^{\text {nd }}$ transmembrane region. It is possible that the Leu46 to polar His substitution affects the membrane spanning domain and the interaction with other transmembrane domains of NS2A or other membrane-associated viral proteins. A change in membrane associated protein topology could lead to a defect in the replication properties of NS2A such as the formation of the replication complex by binding with viral RNA and other components of the replication 
complex.

NS5 is the largest $(104 \mathrm{kDa})$ of the flavivirus proteins, and three functional domains have been identified in NS5: a S-adenosylmethionine methyltransferase-like domain in the N-terminal region (Egloff et al., 2002; Koonin, 1993; Ray et al., 2006), a centrally located nuclear localization sequence (NLS) (Forwood et al., 1999; Kapoor et al., 1995), and an RNA-dependent RNA polymerase (RdRp) domain in the C-terminal region (Bartholomeusz and Wright, 1993; Koonin, 1991). The Asp836 residue in NS5 is located in the RdRp domain. In the homology model of OHFV RdRp domain based on the crystal structure of the dengue virus RdRp domain, this residue is within the $\alpha 25$ helix in the Thumb domain (Yap et al., 2007). In position 836 of the NS5 RdRp domain, negatively-charged amino acids aspartic acid or glutamic acid are highly conserved among most flaviviruses. The molecular model suggests that the negatively-charged Asp836 might form a salt bridge with the positively-charged Lys720 in Motif E of the RdRp domain (Fig. 7). Molecular mechanics calculations also showed that the Asp836 to Gly substitution significantly reduced the interaction of the residue at position 836 with the Lys720 and Motif E (Supplementary Table1). These results led to the hypothesis that the reduction of interaction due to Asp836 to Gly substitution causes structural fluctuation, especially in Motif E. Motif E forms an antiparallel $\beta$-sheet wedged between the palm domain and several $\alpha$-helices of the thumb domain. In several studies, it has been shown that some of the residues in Motif E are involved in the GTP-binding site and which have essential roles in de novo initiation of RNA synthesis in Flaviviridae polymerases (Choi et al., 2004; Lai et al., 1999; Yap et al., 2007). It is possible that residue Asp836 is important for the structural stability of Motif E, and that the structural fluctuation of Motif E caused by the Asp836 to Gly substitution leads to a reduction in the efficiency of de novo initiation of RNA synthesis. In general, the conformational fluctuation of proteins is associated with their temperature. Therefore, this thermal fluctuation could be a possible cause of the temperature-sensitive property observed in the replicon studies (Fig 5). Temperature dependence has been reported at the initiation, 
but not elongation, phase of de novo RNA synthesis by dengue virus RdRp (Ackermann and Padmanabhan, 2001). These data support the involvement of the interaction between Asp836 and Motif E in de novo initiation of RNA synthesis.

In summary, we have constructed an infectious cDNA clone of OHFV and demonstrated the utility of this clone in the research of OHFV pathogenesis. We have also identified previously unknown mutations in NS2A and NS5 that appear to play important roles in OHFV RNA synthesis.

\section{ACKNOWLEDGMENTS}

This work was supported by Overseas Assignment Program for Japanese Researchers in Research into Emerging and Re-emerging Infectious Diseases from The Japan Health Sciences Foundation and Grants-in-Aid for Scientific Research from the Ministry of Education, Science, Sports, and Culture of Japan, Health Sciences Grants for Research on Emerging and Re-emerging Infectious Disease from the Ministry of Health, Labour, and Welfare of Japan, and a Western Regional Center of Excellence (U54 AI057156) Career Development award (MRH) in part.

Ackermann, M. and Padmanabhan, R. (2001) De novo synthesis of RNA by the dengue virus RNA-dependent RNA polymerase exhibits temperature dependence at the initiation but not elongation phase. J Biol Chem 276(43), 39926-37.

Bartholomeusz, A.I. and Wright, P.J. (1993) Synthesis of dengue virus RNA in vitro: initiation and the involvement of proteins NS3 and NS5. Arch Virol 128(1-2), 111-21.

Bredenbeek, P.J., Kooi, E.A., Lindenbach, B., Huijkman, N., Rice, C.M. and Spaan, W.J. (2003) A stable full-length yellow fever virus cDNA clone and the role of conserved RNA elements in flavivirus 
replication. J Gen Virol 84(Pt 5), 1261-8.

Buchen-Osmond, C. (2003) 00.026.0.01.046.02. Tick-borne encephalitis virus group. In: ICTVdB - The

Universal Virus Database, version 3. In: C. Buchen-Osmond (Ed), ICTVdB Management, Columbia University, New York, USA.

Burke, D.S. and Monath, T.P. (2001) Flaviviruses. In: D.M. Knipe and P.M. Howley (Eds), Field 痴 virology. 4 ed., pp. 1043-125. Lippincott Williams \& Wilkins, Philadelphia.

Busygin, F.F. (2000) [Omsk hemorrhagic fever--current status of the problem]. Vopr Virusol 45(3), 4-9.

Campbell, M.S. and Pletnev, A.G. (2000) Infectious cDNA clones of Langat tick-borne flavivirus that differ from their parent in peripheral neurovirulence. Virology 269(1), 225-37.

Chambers, T.J., Hahn, C.S., Galler, R. and Rice, C.M. (1990) Flavivirus genome organization, expression, and replication. Annu Rev Microbiol 44, 649-88.

Choi, K.H., Groarke, J.M., Young, D.C., Kuhn, R.J., Smith, J.L., Pevear, D.C. and Rossmann, M.G. (2004) The structure of the RNA-dependent RNA polymerase from bovine viral diarrhea virus establishes the role of GTP in de novo initiation. Proc Natl Acad Sci U S A 101(13), 4425-30.

The PyMol Molecular Graphics System. DeLano Scientific, San Carlos, CA.

Egloff, M.P., Benarroch, D., Selisko, B., Romette, J.L. and Canard, B. (2002) An RNA cap

(nucleoside-2'-O-)-methyltransferase in the flavivirus RNA polymerase NS5: crystal structure and functional characterization. Embo J 21(11), 2757-68.

Eisenberg, D., Luthy, R. and Bowie, J.U. (1997) VERIFY3D: assessment of protein models with three-dimensional profiles. Methods Enzymol 277, 396-404.

Eswar, N., John, B., Mirkovic, N., Fiser, A., Ilyin, V.A., Pieper, U., Stuart, A.C., Marti-Renom, M.A., Madhusudhan, M.S., Yerkovich, B. and Sali, A. (2003) Tools for comparative protein structure modeling and analysis. Nucleic Acids Res 31(13), 3375-80.

Forwood, J.K., Brooks, A., Briggs, L.J., Xiao, C.Y., Jans, D.A. and Vasudevan, S.G. (1999) The 37-amino-acid interdomain of dengue virus NS5 protein contains a functional NLS and inhibitory CK2 site. Biochem Biophys Res Commun 257(3), 731-7.

Gritsun, T.S. and Gould, E.A. (1998) Development and analysis of a tick-borne encephalitis virus infectious clone using a novel and rapid strategy. J Virol Methods 76(1-2), 109-20.

Gritsun, T.S., Venugopal, K., Zanotto, P.M., Mikhailov, M.V., Sall, A.A., Holmes, E.C., Polkinghorne, I., Frolova, T.V., Pogodina, V.V., Lashkevich, V.A. and Gould, E.A. (1997) Complete sequence of two tick-borne flaviviruses isolated from Siberia and the UK: analysis and significance of the 5' and 3'-UTRs. Virus Res 49(1), 27-39.

Hayasaka, D., Gritsun, T.S., Yoshii, K., Ueki, T., Goto, A., Mizutani, T., Kariwa, H., Iwasaki, T., Gould, E.A. and Takashima, I. (2004) Amino acid changes responsible for attenuation of virus neurovirulence in an infectious cDNA clone of the Oshima strain of tick-borne encephalitis virus. J Gen Virol 85(Pt 4), 1007-18.

Hofmann, K. and Stoffel, W. (1993) TMbase - A database of membrane spanning proteins segments. Biol. 
Chem. Hoppe-Seyler 374, 166.

Holbrook, M.R., Aronson, J.F., Campbell, G.A., Jones, S., Feldmann, H. and Barrett, A.D. (2005) An animal model for the tickborne flavivirus--Omsk hemorrhagic fever virus. J Infect Dis 191(1), 100-8. Kapoor, M., Zhang, L., Ramachandra, M., Kusukawa, J., Ebner, K.E. and Padmanabhan, R. (1995) Association between NS3 and NS5 proteins of dengue virus type 2 in the putative RNA replicase is linked to differential phosphorylation of NS5. J Biol Chem 270(32), 19100-6.

Kharitonova, N.N. and Leonov, Y.A. (1985) Omsk Hemorrhagic Fever. Trans. S. Korzelska. Amerind Publishing Co, New Delhi.

Kinney, R.M., Butrapet, S., Chang, G.J., Tsuchiya, K.R., Roehrig, J.T., Bhamarapravati, N. and Gubler, D.J. (1997) Construction of infectious cDNA clones for dengue 2 virus: strain 16681 and its attenuated vaccine derivative, strain PDK-53. Virology 230(2), 300-8.

Koonin, E.V. (1991) The phylogeny of RNA-dependent RNA polymerases of positive-strand RNA viruses. J Gen Virol 72 ( Pt 9), 2197-206.

Koonin, E.V. (1993) Computer-assisted identification of a putative methyltransferase domain in NS5 protein of flaviviruses and lambda 2 protein of reovirus. J Gen Virol 74 ( Pt 4), 733-40.

Krogh, A., Larsson, B., von Heijne, G. and Sonnhammer, E.L. (2001) Predicting transmembrane protein topology with a hidden Markov model: application to complete genomes. J Mol Biol 305(3), 567-80.

Kummerer, B.M. and Rice, C.M. (2002) Mutations in the yellow fever virus nonstructural protein NS2A selectively block production of infectious particles. J Virol 76(10), 4773-84.

Lai, V.C., Kao, C.C., Ferrari, E., Park, J., Uss, A.S., Wright-Minogue, J., Hong, Z. and Lau, J.Y. (1999) Mutational analysis of bovine viral diarrhea virus RNA-dependent RNA polymerase. J Virol 73(12), 10129-36.

Laskowski, R.A., Macarthur, M.W., Moss, D.S. and Thornton, J.M. (1993) Procheck - a Program to Check the Stereochemical Quality of Protein Structures. Journal of Applied Crystallography 26, 283-291. Leung, J.Y., Pijlman, G.P., Kondratieva, N., Hyde, J., Mackenzie, J.M. and Khromykh, A.A. (2008) Role of nonstructural protein NS2A in flavivirus assembly. J Virol 82(10), 4731-41.

Liu, W.J., Chen, H.B. and Khromykh, A.A. (2003) Molecular and functional analyses of Kunjin virus infectious cDNA clones demonstrate the essential roles for NS2A in virus assembly and for a nonconservative residue in NS3 in RNA replication. J Virol 77(14), 7804-13.

Liu, W.J., Chen, H.B., Wang, X.J., Huang, H. and Khromykh, A.A. (2004) Analysis of adaptive mutations in Kunjin virus replicon RNA reveals a novel role for the flavivirus nonstructural protein NS2A in inhibition of beta interferon promoter-driven transcription. J Virol 78(22), 12225-35.

Liu, W.J., Wang, X.J., Clark, D.C., Lobigs, M., Hall, R.A. and Khromykh, A.A. (2006) A single amino acid substitution in the West Nile virus nonstructural protein NS2A disables its ability to inhibit alpha/beta interferon induction and attenuates virus virulence in mice. J Virol 80(5), 2396-404.

Liu, W.J., Wang, X.J., Mokhonov, V.V., Shi, P.Y., Randall, R. and Khromykh, A.A. (2005) Inhibition of interferon signaling by the New York 99 strain and Kunjin subtype of West Nile virus involves blockage 
of STAT1 and STAT2 activation by nonstructural proteins. J Virol 79(3), 1934-42.

Mackenzie, J.M., Khromykh, A.A., Jones, M.K. and Westaway, E.G. (1998) Subcellular localization and some biochemical properties of the flavivirus Kunjin nonstructural proteins NS2A and NS4A. Virology 245(2), 203-15.

Mandl, C.W., Ecker, M., Holzmann, H., Kunz, C. and Heinz, F.X. (1997) Infectious cDNA clones of tick-borne encephalitis virus European subtype prototypic strain Neudoerfl and high virulence strain Hypr. J Gen Virol 78 ( Pt 5), 1049-57.

Mason, P.W., Bezborodova, S.V. and Henry, T.M. (2002) Identification and characterization of a cis-acting replication element (cre) adjacent to the internal ribosome entry site of foot-and-mouth disease virus. J Virol 76(19), 9686-94.

McElroy, K.L., Tsetsarkin, K.A., Vanlandingham, D.L. and Higgs, S. (2005) Characterization of an infectious clone of the wild-type yellow fever virus Asibi strain that is able to infect and disseminate in mosquitoes. J Gen Virol 86(Pt 6), 1747-51.

Mendez, E., Ruggli, N., Collett, M.S. and Rice, C.M. (1998) Infectious bovine viral diarrhea virus (strain NADL) RNA from stable cDNA clones: a cellular insert determines NS3 production and viral cytopathogenicity. J Virol 72(6), 4737-45.

Munoz-Jordan, J.L., Sanchez-Burgos, G.G., Laurent-Rolle, M. and Garcia-Sastre, A. (2003) Inhibition of interferon signaling by dengue virus. Proc Natl Acad Sci U S A 100(24), 14333-8.

Pletnev, A.G. (2001) Infectious cDNA clone of attenuated Langat tick-borne flavivirus (strain E5) and a 3' deletion mutant constructed from it exhibit decreased neuroinvasiveness in immunodeficient mice. Virology 282(2), 288-300.

Proutski, V., Gould, E.A. and Holmes, E.C. (1997) Secondary structure of the 3' untranslated region of flaviviruses: similarities and differences. Nucleic Acids Res 25(6), 1194-202.

Rauscher, S., Flamm, C., Mandl, C.W., Heinz, F.X. and Stadler, P.F. (1997) Secondary structure of the 3 '-noncoding region of flavivirus genomes: comparative analysis of base pairing probabilities. Rna 3(7), 779-91.

Ray, D., Shah, A., Tilgner, M., Guo, Y., Zhao, Y., Dong, H., Deas, T.S., Zhou, Y., Li, H. and Shi, P.Y. (2006) West Nile virus 5'-cap structure is formed by sequential guanine $\mathrm{N}-7$ and ribose 2 - $\mathrm{O}$ methylations by nonstructural protein 5. J Virol 80(17), 8362-70.

Rossi, S.L., Zhao, Q., O'Donnell, V.K. and Mason, P.W. (2005) Adaptation of West Nile virus replicons to cells in culture and use of replicon-bearing cells to probe antiviral action. Virology 331(2), 457-70. Ruggli, N., Tratschin, J.D., Mittelholzer, C. and Hofmann, M.A. (1996) Nucleotide sequence of classical swine fever virus strain Alfort/187 and transcription of infectious RNA from stably cloned full-length cDNA. J Virol 70(6), 3478-87.

Shi, P.Y., Tilgner, M., Lo, M.K., Kent, K.A. and Bernard, K.A. (2002) Infectious cDNA clone of the epidemic west nile virus from New York City. J Virol 76(12), 5847-56.

Still, W.C., Tempczyk, A., Hawley, R.C. and Hendrickson, T. (1990) Semianalytical Treatment of 
Solvation for Molecular Mechanics and Dynamics. Journal of the American Chemical Society 112(16), 6127-6129.

Tigabu, B., Juelich, T., Bertrand, J. and Holbrook, M.R. (2009) Clinical evaluation of highly pathogenic tick-borne flavivirus infection in the mouse model. J Med Virol 81(7), 1261-9.

Tsui, V. and Case, D.A. (2000) Theory and applications of the generalized Born solvation model in macromolecular simulations. Biopolymers 56(4), 275-91.

Yamshchikov, V., Mishin, V. and Cominelli, F. (2001) A new strategy in design of +RNA virus infectious clones enabling their stable propagation in E. coli. Virology 281(2), 272-80.

Yap, T.L., Xu, T., Chen, Y.L., Malet, H., Egloff, M.P., Canard, B., Vasudevan, S.G. and Lescar, J. (2007) Crystal structure of the dengue virus RNA-dependent RNA polymerase catalytic domain at

1.85-angstrom resolution. J Virol 81(9), 4753-65.

Yoshii, K., Hayasaka, D., Goto, A., Kawakami, K., Kariwa, H. and Takashima, I. (2005) Packaging the replicon RNA of the Far-Eastern subtype of tick-borne encephalitis virus into single-round infectious particles: development of a heterologous gene delivery system. Vaccine 23(30), 3946-56.

Yoshii, K. and Holbrook, M.R. (2009) Sub-genomic replicon and virus-like particles of Omsk hemorrhagic fever virus. Arch Virol 154(4), 573-80.

Yoshii, K., Konno, A., Goto, A., Nio, J., Obara, M., Ueki, T., Hayasaka, D., Mizutani, T., Kariwa, H. and Takashima, I. (2004) Single point mutation in tick-borne encephalitis virus prM protein induces a reduction of virus particle secretion. J Gen Virol 85(Pt 10), 3049-58.

Yun, S.I., Kim, S.Y., Rice, C.M. and Lee, Y.M. (2003) Development and application of a reverse genetics system for Japanese encephalitis virus. J Virol 77(11), 6450-65. 


\section{Figure legends}

Figure 1

Construction of the full-length infectious clone of OHFV. Six cDNA fragments synthesized by RT-PCR were assembled to form the full-length cDNA clone of OHFV (OHF-IC). Restriction sites used to construct OHF-IC are shown at the bottom. A silent mutation (shown in uppercase) was engineered to create a Cla I site $\left(^{*}\right)$. The complete OHFV cDNA is positioned under the control of the T7 promoter. A hepatitis delta virus ribozyme (HDV-RZ)/bacteriophage T7 terminator fragment was fused to the 3' end of the viral genome (see Materials and Methods).

Figure 2

Schematic representation of the genome of OHFV showing all of the amino acid coding difference between strain Guriev and constructed full-length clones (OHF-IC) and OHFV replicons used to analyze the effect of NS2A and NS5 mutations. Bold type has been used to designate the amino acids of the consensus sequence in Guriev (see text and Table 1).

Figure 3

Immunofluorescence staining of BHK cells transfected with the in vitro transcript of OHF-IC-pt (A) and OHF-IC-ori (B). Cells were fixed at 5 days post-transfection and stained with MAb 1H4.

Figure 4

Growth curves of parental Guriev, OHF-IC-pt, OHF-IC-NS5 ${ }_{65}$, OHF-IC-NS5 ${ }_{836}$, OHF-IC-NS5 ${ }_{65-836}$, and OHF-IC-NS2A 4 . A Monolayer of BHK cells was infected with the individual viruses at a multiplicity of infection (MOI) of 0.01. At each time point, the media was harvested and virus titers were determined by plaque assay in BHK cells.

\section{Figure 5}

Effect of NS mutations on OHFV RNA replication. BHK cells were transfected with luciferase-expressing OHFV subgenomic replicon RNAs (OHF-REP-luc) with or without the NS2A or NS5 mutations, and incubated at $37^{\circ} \mathrm{C}$ or $30^{\circ} \mathrm{C}$. Luciferase activities were measured at $6 \mathrm{~h}$ (A) 72 h (B) post-transfection. Luciferase activities are expressed in Raw Light Units (RLU). Asterisks show the statistically significant difference compared to OHF-REP-luc-pt by Student T test $(\mathrm{P}<0.05)$.

\section{Figure 6}

Survival of mice inoculated with OHF-IC. Mice were inoculated subcutaneously with 1,000 p.f.u. of OHF-IC-pt, OHF-IC-NS5 65 , OHF-IC-NS5 ${ }_{836}$, OHF-IC-NS565-836, and OHF-IC-NS2A46. 


\section{Figure 7}

Location of amino acid substitutions at Asp836 on a homology model of the OHFV

RNA-dependent RNA polymerase domain in NS5 based on that of dengue virus (PDB 2J7W). The zoomed region in the right panel shows the proposed interaction of charged side chains between Asp836 and Lys720. 
Figure 1

\section{OHF-IC}

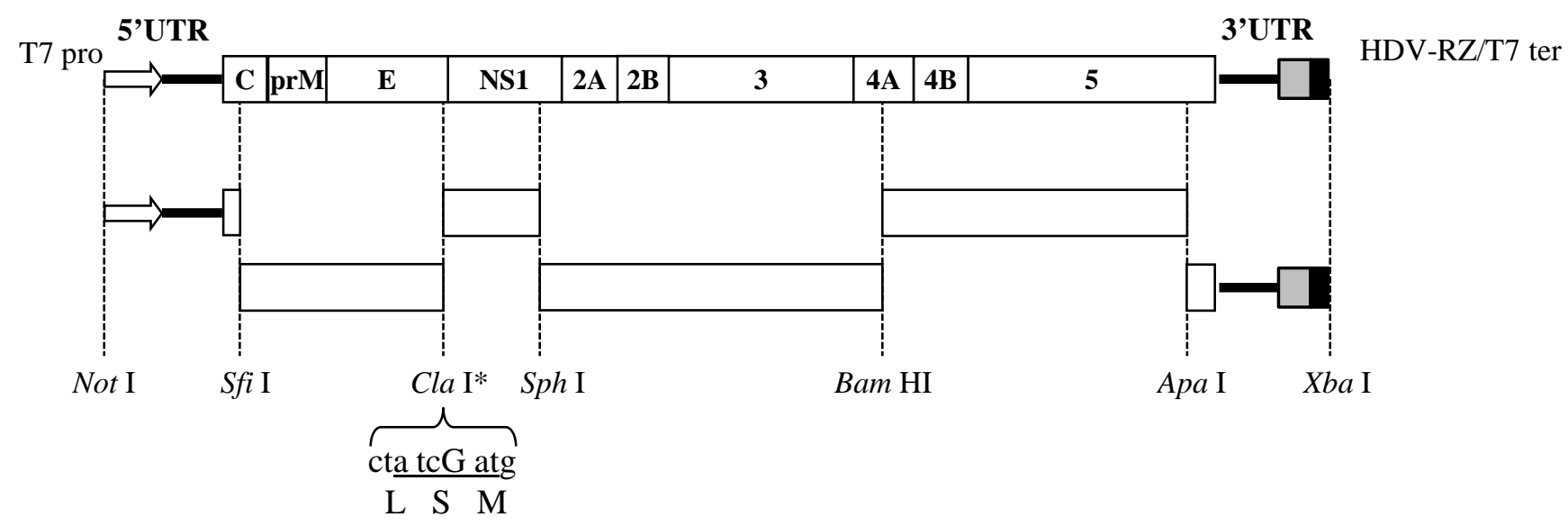


OHF-IC

\begin{tabular}{|l|l|l|l|l|l|l|l|l|l|}
\hline C & prM & E & NS1 & 2A & 2B & 3 & $4 A$ & $4 B$ & 5 \\
\hline
\end{tabular}

OHF-REP-luc

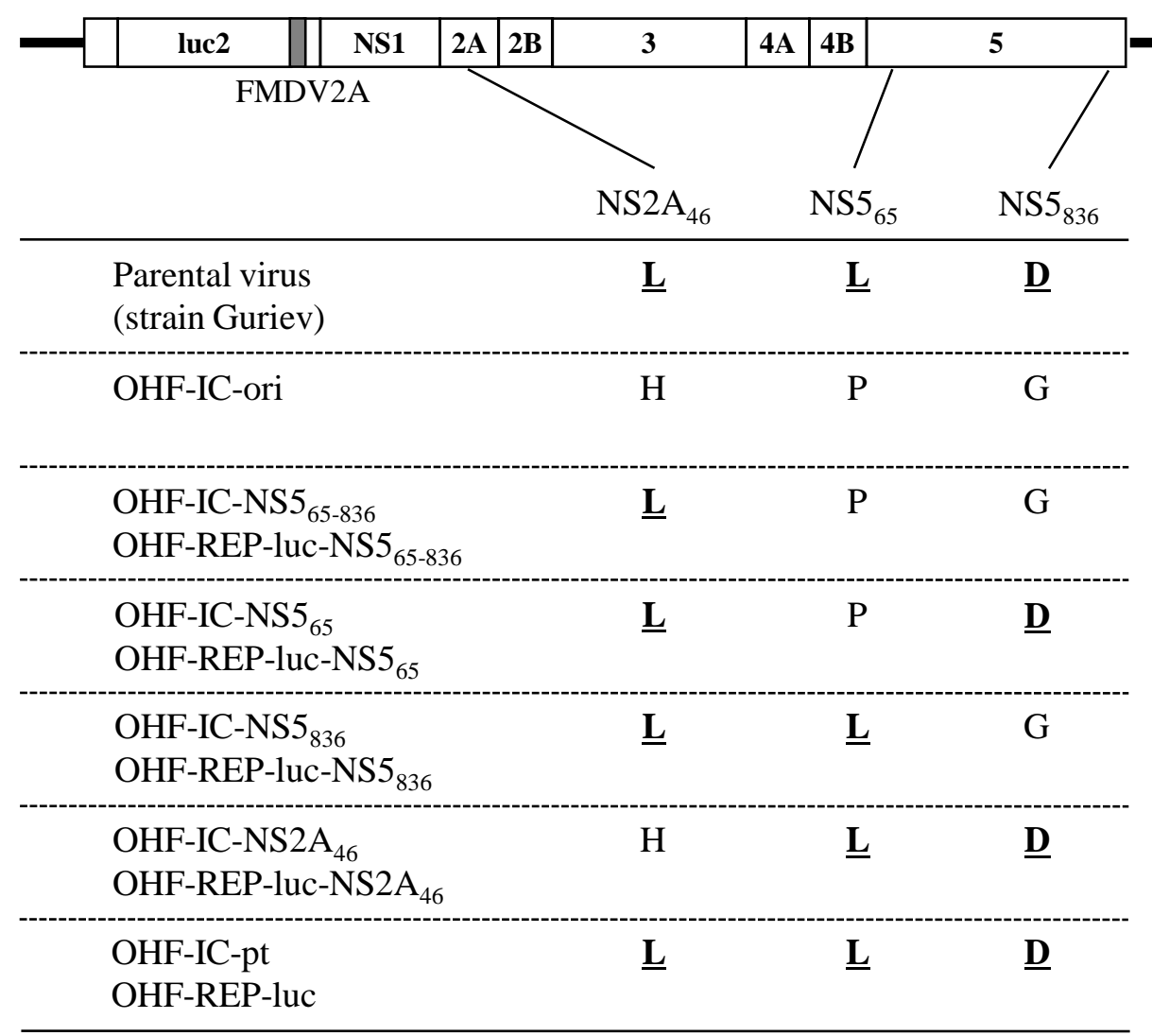


A. OHF-IC-pt

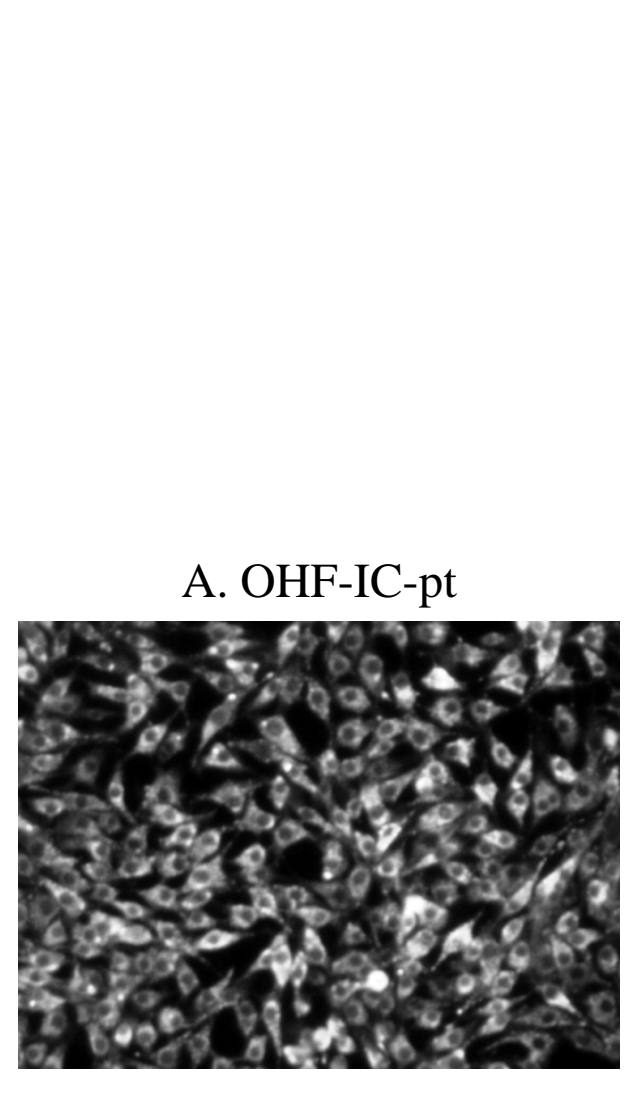

B. OHF-IC-ori

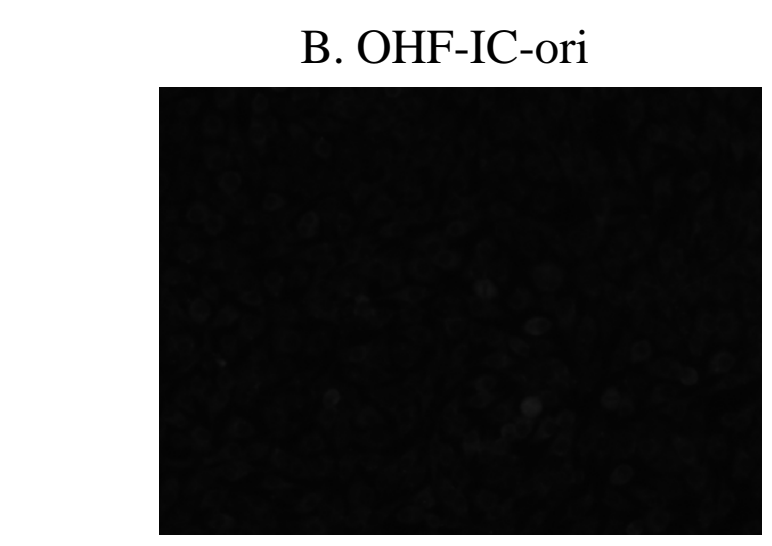

\section{Figure 3}

西

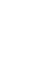

\section{3}




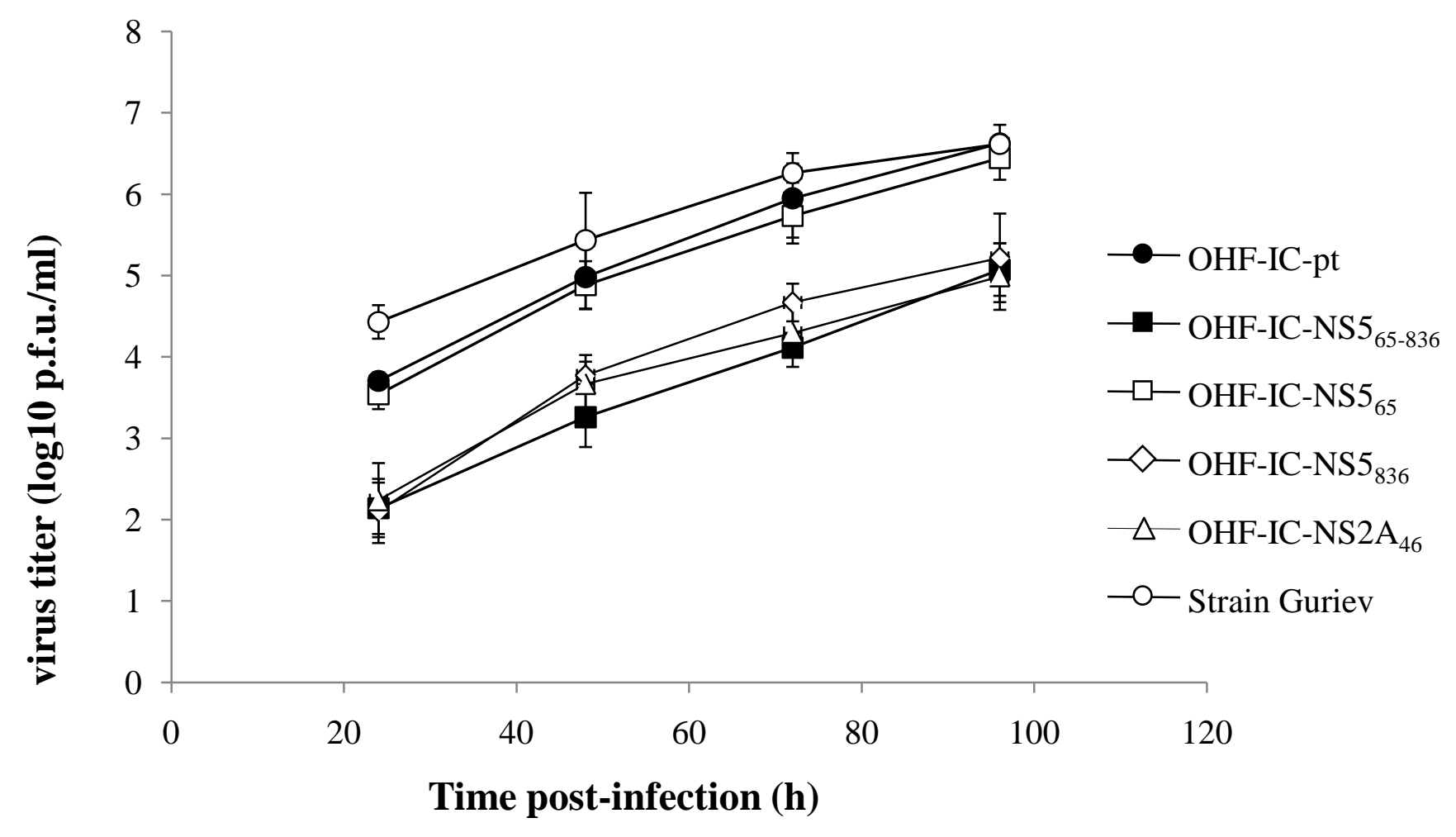


A) 6 hour

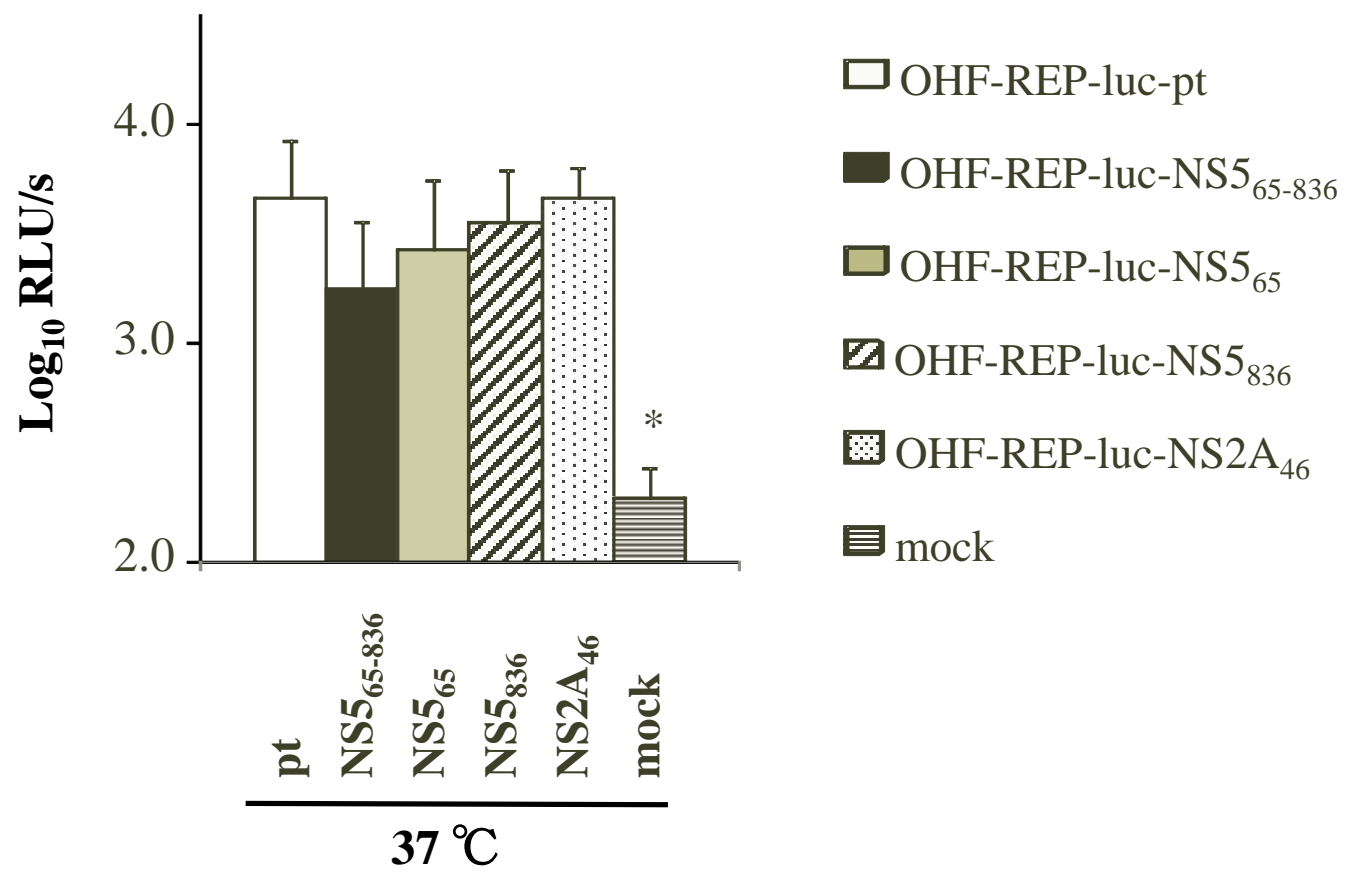


B) 3 day

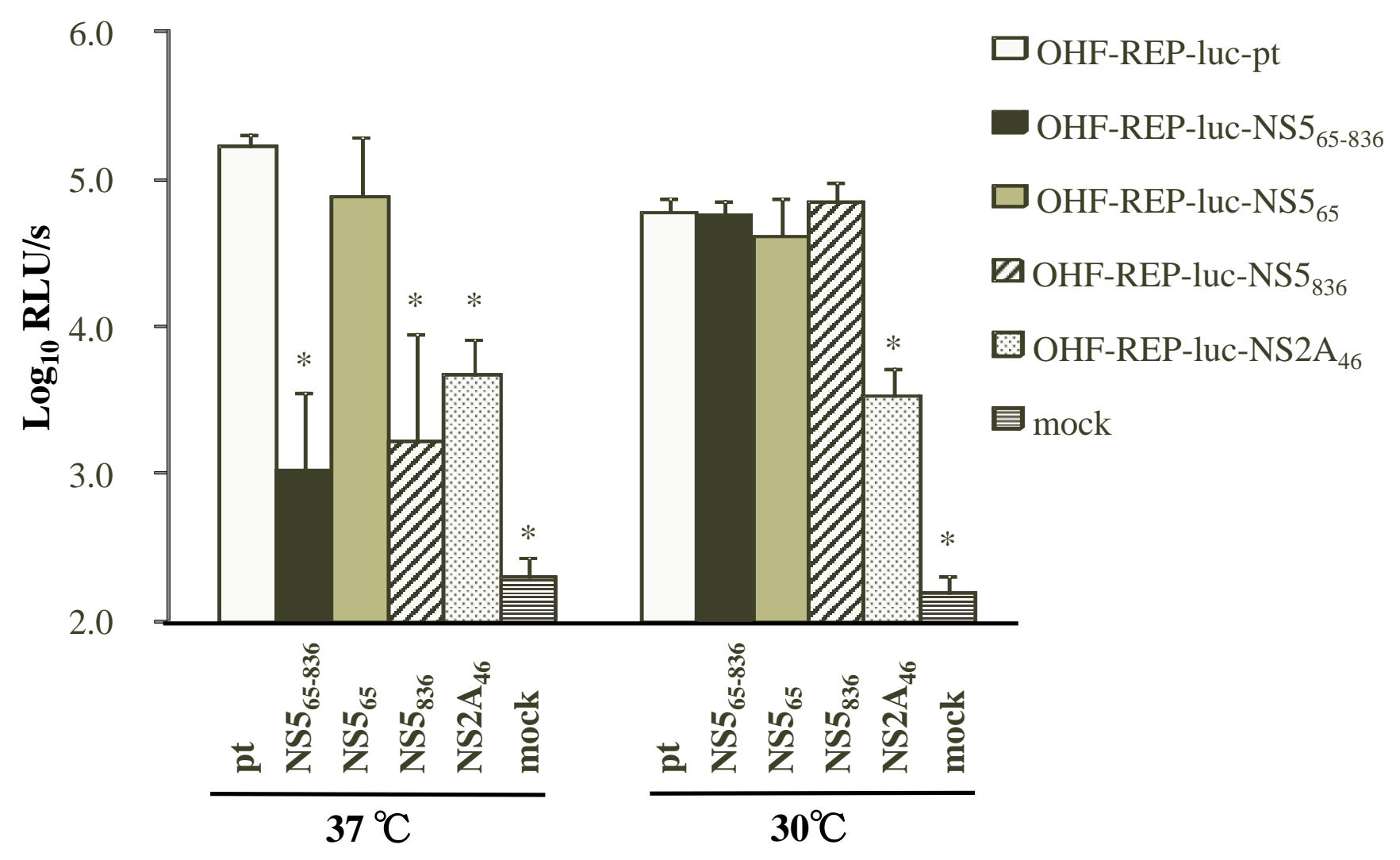




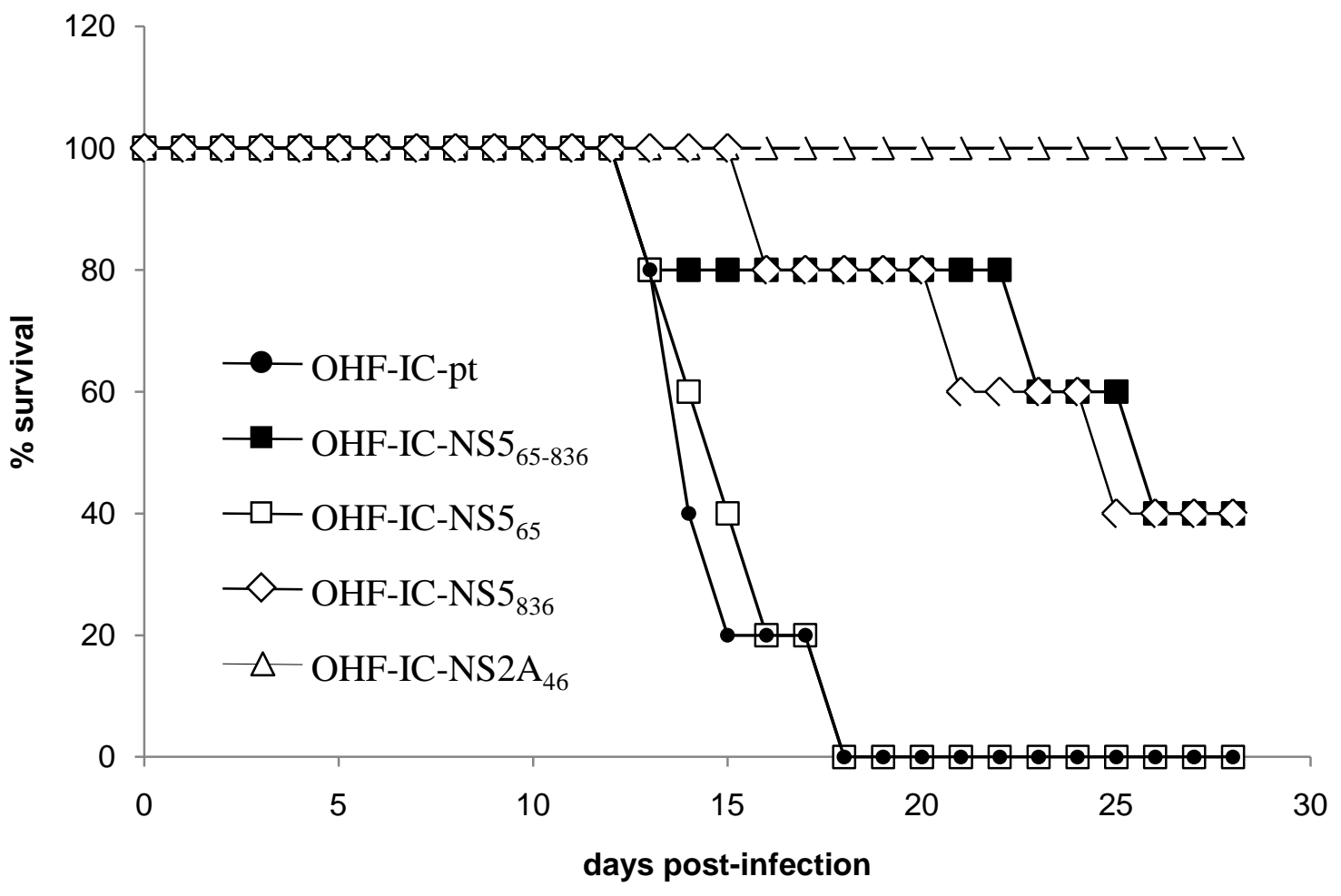




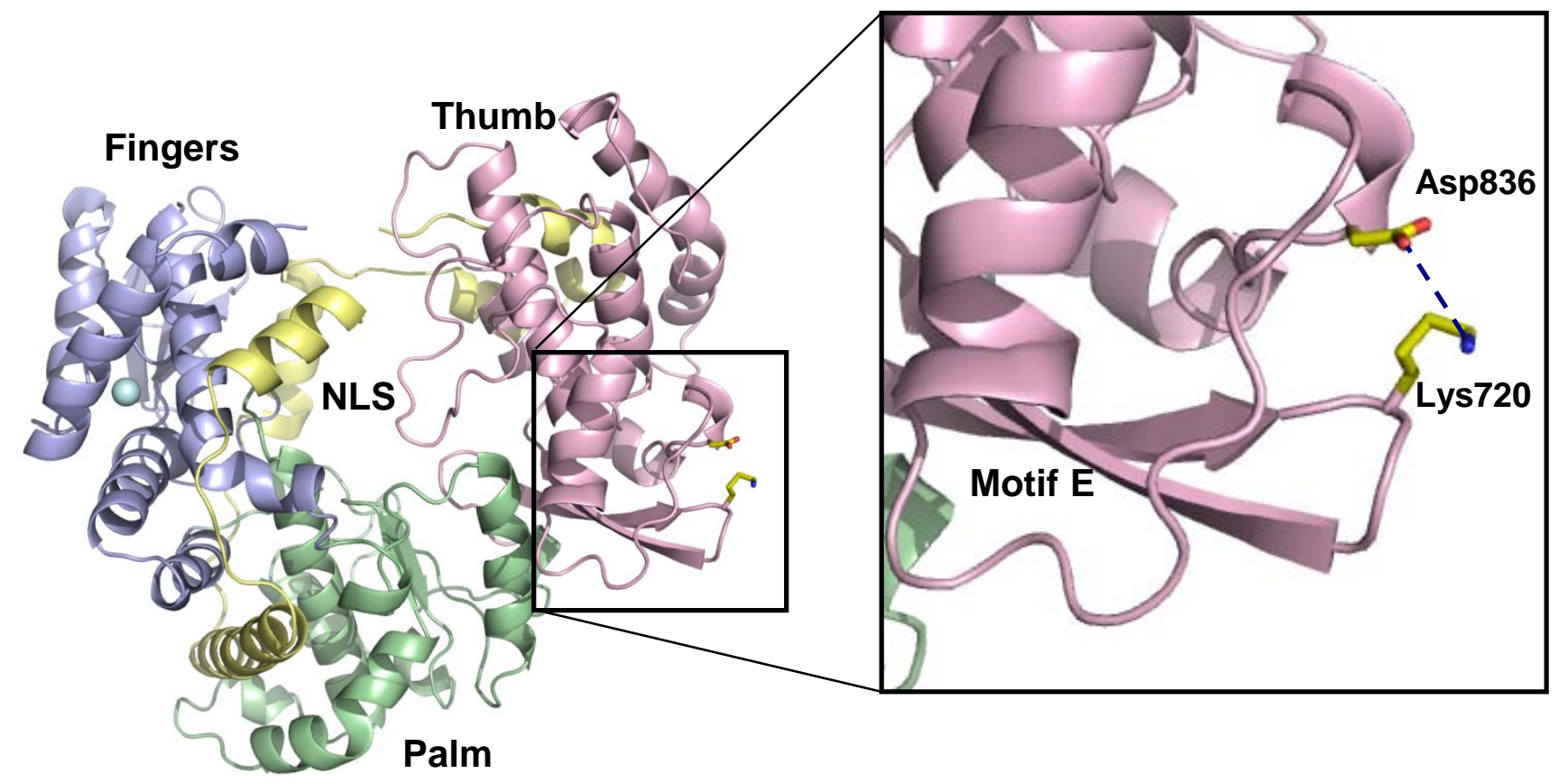


Table 1. Summary of sequence difference between the infectious cDNA OHF-IC-ori and parental OHFV strain Guriev

\begin{tabular}{ccccc}
\hline Base position $^{\text {a }}$ & Strain Guriev & OHF-IC-ori & $\begin{array}{c}\text { Amino } \\
\text { acid }\end{array}$ & Location \\
\hline 297 & A & G & - & \\
2367 & G & A & - & \\
2439 & $\mathrm{C}$ & $\mathrm{G}^{\mathrm{b}}$ & - & \\
3653 & $\mathrm{~T}$ & $\mathrm{~A}$ & $\mathrm{~L} \rightarrow \mathrm{H}$ & NS2A $_{46}{ }^{\mathrm{c}}$ \\
6231 & $\mathrm{C}$ & $\mathrm{T}$ & - & \\
6591 & $\mathrm{~A}$ & $\mathrm{~T}$ & - & \\
6678 & $\mathrm{~T}$ & $\mathrm{C}$ & - & \\
7859 & $\mathrm{~T}$ & $\mathrm{C}$ & $\mathrm{L} \rightarrow \mathrm{P}$ & $\mathrm{NS5}_{65}$ \\
7983 & $\mathrm{~A}$ & $\mathrm{G}$ & - & \\
10172 & $\mathrm{~A}$ & $\mathrm{G}$ & $\mathrm{D} \rightarrow \mathrm{G}$ & $\mathrm{NS5}_{836}$ \\
\hline
\end{tabular}

\footnotetext{
${ }^{a}$ Nucleotide position and sequence are based on OHFV strain Guriev (Accession no. AB507800)

${ }^{\mathrm{b}}$ This silent mutation (shown in uppercase) was engineered to create a Cla I site $\left({ }^{*}\right)$.

${ }^{\mathrm{c}}$ The numbers indicate the amino acid position in each protein
} 